\title{
Leriche Syndrome
}

National Cancer Institute

\section{Source}

National Cancer Institute. Leriche Syndrome. NCI Thesaurus. Code C34773.

An atherosclerotic disorder of the peripheral vascular system affecting mostly males in their later decades. It is caused by thrombotic occlusion of the abdominal aorta just above the level of the bifurcation. Clinical signs include impotence, intermittent claudication, diminished femoral pulses and cold, pallid lower extremities. Prognosis is favorable with surgical or endovascular intervention. 